\title{
A atuação da enfermagem na assistência ao portador de esquizofrenia no ambiente
}

\section{familiar}

\author{
Nursing's performance in assisting schizophrenia patients in the family environment
}

Desempeño de la enfermería en la asistencia a los pacientes con esquizofrenia en el entorno familiar

Recebido: 28/05/2021 | Revisado: 04/06/2021 | Aceito: 08/06/2021 | Publicado: 22/06/2021

Kelly Cristina Spagolla

ORCID: https://orcid.org/0000-0001-7635-0697 Faculdade Cristo Rei, Brasil

E-mail: kellycripagolla@gmail.com

Marli de Oliveira Costa

ORCID: https://orcid.org/0000-0002-9616-4477 Faculdade Cristo Rei, Brasil

E-mail: marli@faccrei.edu.br

\begin{abstract}
Resumo
Entende-se que a saúde mental nos dias atuais não é mais vista como antigamente, principalmente com a chegada da Reforma Psiquiátrica contendo diversos dispositivos para se trabalhar com pacientes portadores de transtornos mentais. Com isso, a assistência profissional dos enfermeiros dada ao paciente esquizofrênico é essencial para atende los, tornando-se um elo entre ele, os profissionais da equipe de saúde e seus familiares. O objetivo geral deste estudo é conhecer de que forma a prestação de assistência da enfermagem no atendimento de pessoas esquizofrênicas pode contribuir na relação com a família durante o processo do tratamento. A metodologia utilizada nesta pesquisa foi uma revisão sistemática de literatura e para isso utilizou-se bases de dados eletrônicos Lilacs, Medline, Scielo e Google Acadêmico. Foram escolhidos artigos publicados entre 2012 a 2020 e selecionados 10 artigos. O estudo possibilitou identificar o papel da equipe de enfermagem na relação com os familiares de portadores de Esquizofrenia, destacando a atribuição do enfermeiro na conscientização dos familiares sobre o tratamento do portador desta doença mental. Orientar as famílias para receber e cuidar do doente mental no ambiente familiar demonstrando uma relação de confiança, relação interpessoal e reabilitação psicossocial. Conclui-se então que a abordagem do enfermeiro a pacientes portadores de esquizofrenia e seus familiares é peça imprescindível no acompanhamento e tratamento, visando melhor qualidade do atendimento e tem papel fundamental como mediador. Torna-se importante realizar novos estudos que possam contribuir com a instrumentalização e qualificação da assistência de enfermagem em direcionar os cuidados com o paciente acometido pela esquizofrenia enfatizando a importância da relação terapêutica entre enfermeiro, paciente e familiares.
\end{abstract}

Palavras-chave: Cuidados de enfermagem; Saúde mental; Esquizofrenia; Família e esquizofrenia.

\begin{abstract}
It is understood that mental health nowadays is no longer seen as it used to be, especially with the arrival of the Psychiatric Reform containing several devices to work with patients with mental disorders. Thus, the professional assistance of nurses given to schizophrenic patients is essential to assist them, becoming a link between them, the professionals of the health team and their families. The general objective of this study is to know how the provision of nursing assistance in the care of schizophrenic people can contribute to the relationship with the family during the treatment process. The methodology used in this research was a systematic review of the literature and for that purpose electronic databases Lilacs, Medline, Scielo and Google Scholar were used. Articles published between 2012 and 2020 were chosen and 10 articles were selected. The study made it possible to identify the role of the nursing team in the relationship with relatives of people with Schizophrenia, highlighting the nurse's role in raising family awareness about the treatment of patients with this mental illness. Guide families to receive and care for the mentally will in the family environment, demonstrating a relationship of trust, interpersonal relationship and psychosocial rehabilitation. It is concluded, then, that the nurse's approach to patients with schizophrenia and their families is an essential part in the monitoring and treatment, aiming at better quality of care and has a fundamental role as a mediator. It is important to carry out new studies that can contribute to the instrumentalization and qualification of nursing care in directing care for patients affected by schizophrenia, emphasizing the importance of the therapeutic relationship between nurses, patients and family members.
\end{abstract}

Keywords: Nursing care; Mental health; Schizophrenia; Family and schizophrenia.

\section{Resumen}

Se entiende que la salud mental hoy en día ya no se ve como antes, especialmente con la llegada de la Reforma Psiquiátrica que contiene varios dispositivos para trabajar con pacientes con trastornos mentales. Así, la assistência 
profesional de enfermeras que se brinda al paciente esquizofrénico es fundamental para atenderlo, convirtiéndose em un nexo entre ellos, los profesionales del equipo de salud y sus familias. El objetivo general de este estudio es conocer cómo la prestación de asistencia de enfermería en el cuidado de personas esquizofrénicas puede contribuir a la relación con la familia durante el proceso de tratamiento. La metodología utilizada en esta investigación fue uma revisión sistemática de la literatura y para ello se utilizaron las bases de datos electrónicas Lilacs, Medline, Scielo y Google Scholar. Se eligieron artículos publicados entre 2012 y 2020 y se seleccionaron 10 artículos. El estúdio permitió identificar el papel del equipo de enfermería en la relación con los familiares de personas con esquizofrenia, destacando el papel de la enfermera en la sensibilización familiar sobre el tratamiento de los pacientes con esta enfermedad mental. Orientar a las familias para recibir y cuidar al enfermo mental en el entorno familiar, demostrando una relación de confianza, relación interpersonal y rehabilitación psicosocial. Se concluye, entonces, que el acercamiento del enfermero a los pacientes con esquizofrenia y sus familias es parte fundamental en el seguimiento y tratamiento, apuntando a una mejor calidad de la atención y tiene un papel fundamental como mediador. Es importante realizar nuevos estudios que puedan contribuir a la instrumentalización y calificación del cuidado de enfermería en el cuidado directo de los pacientes afectados por esquizofrenia, enfatizando la importancia de la relación terapéutica entre enfermeros, pacientes y familiares.

Palabras clave: Atención de enfermería; Salud mental; Esquizofrenia; Familia y esquizofrenia.

\section{Introdução}

A psiquiatria é um dos ramos da medicina que diagnostica e trata de desordens da saúde mental de cunho orgânico e funcional, com manifestações psicológicas severas (Porto, 2021). Diversos são os fatores que podem influenciar a saúde mental de uma pessoa e esses problemas precisam ser tratados antes que causem o rompimento severo às famílias, aos relacionamentos e às comunidades.

A denominação Doença Mental é utilizada para descrever uma alteração na cognição e afetividade das pessoas, que se traduz em perturbações em nível do raciocínio, comportamento, compreensão da realidade e da adaptação às condições da vida tornando os indivíduos acometidos por essas doenças fiquem vulneráveis e ter sintomas que podem variar da confusão mental à uma agitação intensa (de Andrade et al., 2009). Essas são consideradas como uma condição de anormalidade na ordem psíquica, mental ou cognitiva, e podem ter causas determinadas ou não, afetando a vida pessoal, profissional e social das pessoas. Pode-se citar a depressão, o transtorno afetivo bipolar, a esquizofrenia e outras psicoses, demência, deficiência intelectual e transtornos de desenvolvimento, incluindo o autismo.

De acordo com a $\mathrm{OPAS}^{1}$ são vários as doenças mentais e se apresentam de maneiras diferentes e quando estas alterações causam angústia significativa alteram seus pensamentos, emoções ou comportamentos anormais que podem afetar as relações com outras pessoas. Estima-se globalmente que quase 1 bilhão de pessoas vivem com transtorno mental, sendo a depressão a principal causa de incapacidade (OPAS, 2021).

No Brasil, 18,6 milhões de pessoas sofrem de algum transtorno de ansiedade e que, muitas vezes, as pessoas não buscam por tratamentos, e por isso esses números podem ser ainda maiores, desencadeando uma série de outras doenças capazes de inibir e afetar interações sociais (Estadão Saúde, 2020).

As doenças de transtornos mentais vêm crescendo em todos os países do mundo causando impactos sociais, de direitos humanos e econômicos significativos sobre a saúde. No próximo tópico serão aprofundadas informações específicas sobre a esquizofrenia que é o foco da pesquisa.

De acordo com a OMS a esquizofrenia é conhecida como uma doença mental crônica grave e desafiadora e os sintomas são variados e conhecê-los é muito importante para que haja um diagnóstico precoce e um tratamento efetivo desse transtorno. Esta doença atinge cerca de 1\% da população e se inicia antes dos 25 anos de idade, persiste por toda vida e afeta pessoas de todas as classes sociais (Sadock et al., 2007). As primeiras manifestações surgem, geralmente, entre o fim da

\footnotetext{
${ }^{1}$ Organização Pan Americana de Saúde
} 
adolescência e o início da idade adulta, e costuma ser notada sua presença após um episódio psicótico, pois não existe um teste específico para ser feito, por isso não se sabe como preveni-la.

No tratamento da esquizofrenia vem ocorrendo algumas mudanças no conceito de doença mental e assistência na área, pois agora o foco não é apenas no aspecto biológica, mas no psicossocial também. Essa nova concepção atua em verificar não somente a doença, mas em qualidade de vida ao portador de esquizofrenia (Oliveira et al., 2012).

O tratamento da esquizofrenia se molda em três componentes principais: terapêutico, para alívio de sintomas e recidiva; educação e intervenções psicossociais aos doentes e suas famílias, evitando recorrências e reabilitar os doentes a reintegrarem-se na comunidade e a recuperarem o funcionamento social, educacional e ocupacional (A. M. P. Cardoso, 2008).

Nesse sentido, o tratamento farmacológico é essencial, visto que permite obter melhores resultados principalmente quando há combinação com a intervenção psicossocial pode minimizar o impacto de acontecimentos derivados do contexto em que a pessoa doente vive o que pode ter um efeito eficaz no controle da esquizofrenia. Esse tipo de tratamento não tem um tempo determinado, e depende de um estudo e metas análises de acordo a cada paciente e estudos mostram que $25 \%$ dos pacientes com um bom tratamento tem tido boa qualidade de vida (Holmes, 1997).

Por isso é importante que os integrantes da família, por serem muitas vezes, o único meio de contato social com os esquizofrênicos deve mostrar interesse e transmitir confiança a eles, para obter bons resultados no tratamento.

Portanto, é de extrema importância que os assistentes de enfermagem conheçam com profundidade os sintomas e formas de tratamento de pacientes esquizofrênicos para atender suas necessidades e ajudá-los. Assessorar seus familiares também é primordial, pois quando essa doença é bem compreendida, há grande melhora na qualidade de vida do portador desta enfermidade.

A Enfermagem é uma profissão que tem como essência, cuidar do ser humano nos aspectos biológicos, psicológicos, sociais e culturais, acompanhando-os no processo das doenças, tendo em vista a promoção, prevenção e recuperação da saúde das pessoas. A função do enfermeiro é cuidar do outro e, dentro da proposta de área de Saúde Mental não é diferente. Este profissional deve ter conhecimento para lidar com cada situação de doença, e não perder de vista o compromisso terapêutico e juntamente com a equipe interdisciplinar, tem grande desafio ao trabalhar com portadores de sofrimento mental grave, como os esquizofrênicos.

O enfermeiro atua diariamente na promoção e preservação da saúde mental, fazendo intervenções quando necessário nos diversos níveis, seja de leve a grave, agudos e crônicos, uma vez que os portadores de esquizofrenia, por se limitarem a cuidar de si mesmos (Stefanell et al., 2008). A atuação da enfermagem tem papel importante no processo de reabilitação dos sujeitos esquizofrênicos e objetiva a promoção de cuidar integralmente em saúde mental abrangendo as distintas fases dos transtornos, bem como os diferentes níveis de atenção à saúde.

$\mathrm{O}$ enfermeiro e sua equipe são peça fundamental no processo de humanização e precisam dirigir suas ações no atendimento das necessidades do esquizofrênico visando melhorar a qualidade do atendimento, na busca de valorizar os hábitos e cultura em todas as fases do tratamento (Castro \& Furegato, 2008)

Percebe-se que existe atualmente novas exigências no trabalho dos enfermeiros, voltadas no resgate da subjetividade e todo o contexto de vida dos pacientes, ou seja, a comunicação desenvolvida no enfermeiro sendo capaz de aproximar-se das pessoas ao seu redor no desenvolvimento de suas atividades profissionais. O uso de novos modelos de enfermagem e teorias para o paciente de planejamento e de saúde cuidados podem oferecer melhores serviços para indivíduo e da comunidade (A. Santos, 2017).

Nesse sentido é necessário que o profissional de enfermagem possa refletir sobre o processo de cuidar do paciente esquizofrênico de modo sistêmico, que permite dar assistência e aplicar os conhecimentos teóricos que podem direcionar e 
embasar suas ações. Essa assistência deve estabelecer uma relação eficaz o que requer dos profissionais, grande cuidado no contato inicial, pois estão em situação de conflito e com necessidade de falar sobre suas angústias (Videbeck. Sheila L., 2012).

Assim sendo, esse momento é primordial que o enfermeiro saiba abordar o paciente esquizofrênico por meio de palavras diretas, claras e simples, questionando-o com cuidado para trazê-los para a realidade e ajudá-los a utilizar seus pontos fortes e qualidades perante determinadas situações, dando-lhes feedback positivo para deixá-los autoconfiantes no tratamento.

Outro ponto muito sensível é o cuidado que os profissionais de enfermagem devem ter com a família do doente, pois são eles que acompanham diretamente a sua evolução no meio social onde vivem e podem contribuir para uma melhor articulação com a comunidade possibilitando que esse paciente se sinta incluso na sociedade.

A família é composta por um grupo de pessoas que possui vínculos afetivos, cujos laços são baseados na confiança e no suporte mútuo, e tem como função cuidar, apoiar, incentivar, e estar presente na vida de todos os seus integrantes. Quando um membro da família adoece causa grande preocupação, principalmente em situação de doença de saúde mental, como a esquizofrenia, no qual todos os seus integrantes passam a lidar com vários sintomas ainda desconhecidos que podem desestabilizar a estrutura familiar. Ficam impactados e se sentem desprotegidos, apresentando ansiedade e insegurança por não saberem lidar com os comportamentos ora apresentados, e qual a melhor maneira de cuidar do paciente.

A participação da família é fundamental na recuperação do portador com esquizofrenia, sendo uma parceira importante dos profissionais de enfermagem no processo de reabilitação psicossocial. Assim, a assistência profissional dos enfermeiros dada ao paciente é essencial para atender e apoiar estas famílias, se tornando um elo entre o paciente esquizofrênico, demais profissionais da equipe de saúde e os familiares, uma vez que estão mais presentes no contato diário com esses indivíduos, compartilhando as dificuldades, sintomas, queixas e comportamentos.

Esta pesquisa buscou contribuir para a melhoria da sociedade, no sentido de conhecer a assistência de enfermagem oferecida a estas famílias no atendimento às necessidades básicas do portador de esquizofrenia. Para a comunidade acadêmica, que traga contribuição científica à sua área de conhecimento, servindo como material de consulta às pessoas que se interessem sobre $\mathrm{o}$ assunto.

Assim, o objetivo desse trabalho foi sobre a atuação da enfermagem no atendimento a pessoas esquizofrênicas, e como a enfermagem pode contribuir na relação com a família durante o processo do tratamento.

\section{Metodologia}

Para construir esse estudo foi realizado um levantamento bibliográfico da literatura a partir de artigos científicos, livros e teses publicadas na internet e outros. A metodologia utilizada foi uma revisão sistemática de literatura que permitiu formular conclusões gerais no que diz respeito à determinada área do conhecimento mediante uma síntese de estudos publicados.

A metodologia é de natureza qualitativa embasada em uma revisão sistemática de literatura com critérios previamente selecionados, a fim de reunir publicações científicas de acordo com o tema esquizofrenia e a atuação da enfermagem na assistência para as famílias no sentido de orientá-las e apoiá-las no enfrentamento do tratamento de pessoas esquizofrênicas, que exige a produção de estudos para compreender o fenômeno. Para coleta dos artigos utilizou-se os descritores "Esquizofrenia", "Cuidados de Enfermagem", "Saúde Mental”, "Família e Esquizofrenia".

A coleta de dados estruturada aconteceu entre os meses de fevereiro a abril de 2021, através de busca avançada, que permitiu interligar descritores para refinar a pesquisa, nas bases de dados: LILACS $^{2}$, MEDLINE $^{3}$, SCIELO $^{4}$ e Google Acadêmico.

${ }^{2}$ Literatura Latino-Americana e do Caribe em Ciências da Saúde 
Como critérios de inclusão foram escolhidos artigos em português, publicados entre 2012 a 2020 que retratam sobre estudos recentes sobre a temática esquizofrenia que dispunham de texto completo. A princípio os artigos foram selecionados por título e resumo com base no atendimento dos critérios de inclusão. Com aplicação de critérios de exclusão baseados na relevância do conteúdo e tema para o trabalho proposto e restaram 10 artigos.

Neste estudo com enfoque na revisão sistemática de literatura foram encontrados os artigos que atendem aos critérios na busca previamente estabelecida, conforme os procedimentos teórico-metodológicos adotados.

\section{Resultados e Discussão}

A psiquiatria nasce no momento em que se incorpora na medicina como nova especialidade e para se falar de saúde mental é necessário compreender ou diferenciar normalidade de anormalidade. Para isso, é preciso conhecer um pouco na história para entender a loucura no mundo.

A história da psiquiatria é de asilamento, no qual a pessoa considerada como louca era afastada do convívio da sociedade e isso se deu em meados do século XVII na Europa, devido à ordem burguesa então em construção (Rodriguez Belmonte, 1996). Nessa representação histórica, o conceito de loucura está intimamente relacionado ao surgimento dos manicômios, chamados posteriormente de hospitais psiquiátricos, ambos estão ligados aos períodos de diferentes épocas.

De acordo com Lopes (Lopes, 2001) a doença mental durante a história da humanidade era considerada como ação sobrenatural na antiguidade pré-clássica e no final da Idade Média até a Idade Moderna era vista como uma pessoa possuída pelo demônio, sendo aprisionada e torturada.

No século XVIII, na França, os doentes mentais foram libertados por Phillippe Pinel, após estudos relacionados a saúde mental e Psiquiatria. A partir disso, os distúrbios da mente passaram a ser tratados como doença e o 'louco' como doente (Fortes, 2010). Phillippe Pinel, considerado como o pai da psiquiatria entendeu que a loucura deveria ser tratada e classificada e nessa época os asilos foram substituídos pelos manicômios.

No final do século XVIII, a psiquiatria passa a ser reconhecida por um saber próprio do tratamento de doentes mentais. Concebeu-se a necessidade de um lugar apropriado e separado para o tratamento de doentes mentais (Sade, 2014).

Segundo Ramminger (2002), a partir do século XIX na Europa as instituições manicomiais passaram a ser uma modalidade terapêutica eficaz, mesmo com o reconhecimento de alguns pontos negativos como o isolamento que eram justificados como um mal necessário. No Brasil como na Europa, os 'loucos' também perambulavam pelas ruas das cidades, e algumas vezes eram recolhidos nas Santas Casas de Misericórdia ou outros hospitais.

Desde a época imperial, as pessoas portadoras de algum transtorno mental eram afastadas do convívio social e colocadas em asilos, principalmente àquelas oriundas de famílias tradicionais e ricas da sociedade carioca (P. A. N. Santos \& Miranda, 2015). Nesse sentido, as famílias tradicionais da época do império tinham 'vergonha' de ter entre seus familiares, pessoas 'loucas' e as afastavam do convívio da sociedade, colocando-as em asilos.

No século XIX no Brasil a psiquiatria científica passou a ser implementada e com isso aconteceram transformações referentes à assistência prestada aos pacientes psiquiátricos. Em 1852 foi inaugurado o primeiro Hospital Psiquiátrico na cidade do Rio de Janeiro para abrigar os 'loucos' da Corte e demais províncias do Império sendo o primeiro manicômio estabelecido no Brasil. Neste hospital ainda não se aplicavam técnicas de tratamentos para doenças mentais e ainda mantinha os maus tratos, além do aspecto de prisão e cárcere.

Nesses manicômios os pacientes internados tinham diversos transtornos mentais e não possuíam direitos como indivíduos e eram tratados com desrespeito. A primeira Lei Federal de Assistência foi promulgada em 1912 que ganhou status

\footnotetext{
${ }^{3}$ Medical Literature Analysis and Retrieval System Online
}

${ }^{4}$ Scientific Electronic Library Online 
de uma especialidade médica autônoma aos médicos psiquiatras, o que aumentou o número de instituições destinadas aos portadores de transtornos mentais. Em 1936, criou-se Instituto de Psiquiatria e, mais tarde, em 1941, o Serviço Nacional de Doenças Mentais (Carniel, 2008).

A saúde mental, em 1970 passou a ser de responsabilidade da Divisão Nacional de Saúde Mental e o tratamento psiquiátrico começou a ser realizado por uma equipe multidisciplinar, com a atuação do psicólogo, assistente social, enfermeiro, auxiliares técnicos e de administração (P. A. N. Santos \& Miranda, 2015). No final da década de 1970, começa a ser feita a reforma psiquiátrica no Brasil juntamente com instituições, poder público e forças de diversos lugares (Souza \& Gusmão, 2017).

Em 2001, promulgou-se a Lei 10.216 da Reforma Psiquiátrica que, trata sobre os direitos da pessoa doente mental, de sua autonomia quanto aos tratamentos propostos, da não internação desnecessária, da internação voluntaria e involuntária, e da reinserção do doente em seu meio social (Brasil, 2001). A reforma psiquiátrica brasileira criou novos dispositivos por meio da inserção das ações de saúde mental na saúde pública, possibilitando novas abordagens, princípios e valores para as pessoas com sofrimento psíquico, o que impulsionou maneiras mais adequadas de cuidado à loucura no seu âmbito familiar, social e cultural.

As mudanças na saúde mental ainda não incluem buscar uma cura, mas sim dar uma assistência de qualidade ao indivíduo que sofre de doenças mentais e necessita receber o melhor tratamento disponível.

Segundo a Classificação Internacional das Doenças a Esquizofrenia é classificada como CID 10 - F20 e se caracteriza como uma enfermidade complexa, por meio de distorções do pensamento, percepção de si mesmo e da realidade externa, além de inadequação e embotamento do afeto (Iacoponi, 1999). Alguns autores atribuem essa doença a diversos fatores biológicos ligados à genética ou anormalidade de estruturas cerebrais e deficiência em neurotransmissores que ao se interagirem, criando situações no qual podem ser favoráveis ou não, ao aparecimento do transtorno (Videbeck. Sheila L., 2012).

A esquizofrenia é uma desordem hereditária, e ter um parente com essa doença é fator de risco significativo para desenvolvê-la e apresenta distorções funcionais em vários graus no qual os processos cognitivos e outras funções dos pacientes com essa síndrome encontram-se alterados.

A esquizofrenia pode ser subdividida em tipos. O primeiro tipo é chamado de paranoide desorganizado, sendo predominado por delírios, alucinações, perturbações do afeto e pragmatismo; o segundo tipo é o hebefrênico, quando há desorganização nos pensamentos, discursos incoerentes, associações a ideias ilógicas. O terceiro tipo é o residual, forma crônica da doença havendo uma progressão clara de sintomas psicóticos (Tengan \& Maia, 2004).

A doença esquizofrenia é um processo doloroso, e vem acompanhada de intenso sofrimento e limitações decorrentes da deterioração de vários processos mentais, e apresenta no indivíduo alguns sintomas característicos conhecidos como sintomas positivos e sintomas negativos. Os sintomas positivos são delírios, alucinações, pensamentos incoerentes, agitação psicomotora e afeto inadequado. Os sintomas negativos são deficiência intelectual e de memória, pobreza de discurso, incapacidade de sentir prazer, isolamento social e falta de motivação (da Silva, 2006).

Muitos são os estudos relacionados a Esquizofrenia realizados e publicados em bases de dados especializados anualmente. Na Tabela 1 são apresentados os trabalhos que constituem o levantamento realizado e estão organizados em ordem crescente de nome de autor. 
Tabela 1 - Elementos e características dos artigos da Revisão

\begin{tabular}{|c|c|c|}
\hline AUTORIA/ ANO & OBJETIVO & RESULTADOS \\
\hline 1- Alves et al., 2018 & $\begin{array}{l}\text { Avaliar os problemas dos cuidadores de } \\
\text { doentes com esquizofrenia em termos de } \\
\text { sobrecarga objetiva e subjetiva. }\end{array}$ & $\begin{array}{l}\text { Nas intervenções que reduzam a sobrecarga } \\
\text { familiar o enfermeiro em saúde mental dos } \\
\text { cuidados de saúde primários tem papel primordial. }\end{array}$ \\
\hline 2- Araújo et al., 2020 & $\begin{array}{l}\text { Analisar os diferentes obstáculos e } \\
\text { dificuldades que uma família enfrenta } \\
\text { quando possui um portador de } \\
\text { esquizofrenia no lar, e como a } \\
\text { enfermagem pode colaborar com o } \\
\text { tratamento e melhoria na qualidade de } \\
\text { vida dos mesmos. }\end{array}$ & $\begin{array}{l}\text { A relação enfermagem e familiares dos } \\
\text { esquizofrênicos são compartilhadas por meio dos } \\
\text { sentimentos e emoções gerados pela experiência } \\
\text { entre eles. estabelecendo uma relação positiva nos } \\
\text { familiares, na busca da melhoria do cuidado ao } \\
\text { paciente. }\end{array}$ \\
\hline $\begin{array}{l}\text { 3- D’Assunção et al., } \\
2016\end{array}$ & $\begin{array}{l}\text { Identificar as representações sociais que } \\
\text { os profissionais de enfermagem possuem a } \\
\text { respeito do relacionamento com os } \\
\text { cuidadores dos portadores de } \\
\text { esquizofrenia }\end{array}$ & $\begin{array}{l}\text { A equipe de saúde pode auxiliar a família e } \\
\text { cuidadores a compreender o transtorno e enfrentar } \\
\text { as demandas, contribuindo, assim, para o } \\
\text { empoderamento. }\end{array}$ \\
\hline $\begin{array}{l}\text { 4- Gomes \& Mello, } \\
2012\end{array}$ & $\begin{array}{l}\text { Analisar o grau de sobrecarga do principal } \\
\text { cuidador que convive com o portador de } \\
\text { esquizofrenia, em um hospital de } \\
\text { emergência psiquiátrica do Estado do Rio } \\
\text { de Janeiro. }\end{array}$ & $\begin{array}{l}\text { Foram encontradas sobrecargas elevadas na rotina } \\
\text { diária do familiar e intenso abalo da sua saúde } \\
\text { mental, sendo fundamental que a enfermagem } \\
\text { inclua as famílias no tratamento, diminuindo as } \\
\text { sobrecargas. }\end{array}$ \\
\hline $\begin{array}{l}\text { 5- Pereira Lopes \& } \\
\text { Buriola, } 2015\end{array}$ & $\begin{array}{l}\text { Realizar um levantamento de textos } \\
\text { nacionais que descreva o conceito a } \\
\text { epidemiologia e o papel da enfermagem } \\
\text { na adesão ao tratamento da esquizofrenia }\end{array}$ & $\begin{array}{l}\text { O enfermeiro tem papel fundamental na } \\
\text { orientação sobre a doença, medicamentos } \\
\text { auxiliando assim a família na convivência com o } \\
\text { paciente e reintegrá-lo à sociedade }\end{array}$ \\
\hline 6- Melo et al., 2020 & $\begin{array}{l}\text { Relatar a experiência de duas acadêmicas } \\
\text { de enfermagem frente a um caso de } \\
\text { esquizofrenia em idosa }\end{array}$ & $\begin{array}{l}\text { Os familiares dos pacientes esquizofrênicos estão } \\
\text { cansados, sobrecarregados e muitas vezes até } \\
\text { mesmo ficando doentes. }\end{array}$ \\
\hline 7- Ruano et al., 2016 & $\begin{array}{l}\text { Identificar os aspectos do tratamento da } \\
\text { esquizofrenia, bem como a contribuição } \\
\text { da enfermagem no enfrentamento pelo } \\
\text { indivíduo e sua família }\end{array}$ & $\begin{array}{l}\text { Os cuidados de enfermagem baseiam-se em } \\
\text { estratégias para a adesão do mesmo ao tratamento. } \\
\text { É papel da equipe de enfermagem orientar a } \\
\text { família e paciente sobre a terapia medicamentosa } \\
\text { e seus efeitos colaterais. }\end{array}$ \\
\hline $\begin{array}{l}\text { 8- Monteiro dos Santos } \\
\text { et al., } 2019\end{array}$ & $\begin{array}{l}\text { Elucidar, por meio de revisão } \\
\text { bibliográfica, aspectos relacionados à } \\
\text { esquizofrenia com vistas à atuação da } \\
\text { enfermagem no tratamento dos pacientes } \\
\text { portadores dessa doença }\end{array}$ & $\begin{array}{l}\text { A equipe de Enfermagem e a equipe } \\
\text { multidisciplinar, tem papel importante na } \\
\text { reabilitação ao paciente que sofre com transtornos } \\
\text { mentais, como a Esquizofrenia. }\end{array}$ \\
\hline $\begin{array}{l}\text { 9- Souza \& Gusmão, } \\
2017\end{array}$ & $\begin{array}{l}\text { Discutir a assistência de enfermagem } \\
\text { prestada ao portador de esquizofrenia, } \\
\text { para tanto, esta trata-se de uma revisão } \\
\text { integrativa da literatura com artigos } \\
\text { datados de } 2011 \text { a } 2017 \text {. }\end{array}$ & $\begin{array}{l}\text { A assistência de enfermagem deve alcançar a } \\
\text { família e as relações interpessoais do portador de } \\
\text { esquizofrenia, no intuito de mantê-lo no convívio } \\
\text { familiar e social, ampliando a sua interação social. }\end{array}$ \\
\hline 10- Vaz, 2014 & $\begin{array}{l}\text { O estudo dos cuidados de enfermagem } \\
\text { dirigidos à família cuidadora da pessoa } \\
\text { com esquizofrenia }\end{array}$ & $\begin{array}{l}\text { Para os familiares cuidadores das pessoas com } \\
\text { esquizofrenia veem o enfermeiro como um mero } \\
\text { executor técnico. }\end{array}$ \\
\hline
\end{tabular}

Fonte: Autores (2021).

Dos artigos selecionados, três estudos realizaram revisão Integrativa de literatura. Duas revisões bibliográficas. Quatro pesquisas descritivas e uma revisão de literatura.

A discussão foi feita em duas etapas; a primeira traz o papel da enfermagem na relação família e portadores da doença, e a segunda etapa discute-se as dificuldades da família frente ao esquizofrênico. 


\subsection{Papel da equipe de enfermagem na relação com os familiares de portadores de Esquizofrenia.}

O Profissional de Enfermagem deve exercer suas atividades com competência para promover o ser humano na sua integralidade, de acordo com os princípios da ética e da bioética. Conhecer a doença esquizofrenia na teoria e prática compreende destacar o papel do enfermeiro na conscientização dos familiares sobre o tratamento do portador desta doença mental.

\section{- Orientação para a Família}

Para Gomes e Mello (2012) e Pereira e Buriola (2015), os profissionais de saúde tem o papel fundamental de orientar a família para receber e cuidar do doente mental no ambiente familiar, de forma que possa enfrentar situações complicadas, advindas da convivência com o portador do sofrimento psíquico devendo trabalhar em conjunto com as famílias, os doentes para detectar o grau de sobrecarga dos principais cuidadores da pessoa adoecida, desenvolvendo propostas de cuidado às famílias, minimizando os encargos familiares e incrementando a qualidade de vida do portador de esquizofrenia, assim como do grupo familiar. Esses profissionais devem auxiliar a família na convivência com o paciente e reintegrá-lo na sociedade, diminuindo assim o preconceito e a discriminação pela falta de conhecimento relacionado à doença.

\section{- Relação de Confiança e Relação Interpessoal}

D' Assunção et al. (2016), Souza e Gusmão (2017) relatam que no relacionamento da enfermagem e familiares são estabelecidas relação de confiança e cumplicidade expondo opiniões, pensamentos, trocas e enfrentamento de problemas. É por meio da comunicação que o familiar expõe suas dúvidas e buscam informações a respeito do paciente e com isso os profissionais de saúde podem ajudar os familiares a se sentirem seguros no cuidado e convivência com o portador de esquizofrenia no ambiente familiar. É muito importante o relacionamento interpessoal na assistência ao portador de esquizofrenia, tanto no lidar com a família quanto no contato direto com o paciente. Sugere-se que a assistência de enfermagem deve alcançar a família e as relações interpessoais no intuito de mantê-lo no convívio familiar e social.

\section{- Reabilitação Psicossocial}

No estudo de Vaz (2014), Ruano; Silva; Aquino (2016); Alves et al. (2018) e; Santos; Marques; (2019), os profissionais de enfermagem devem atuar em cuidados de saúde primários, especialista de saúde mental e psiquiátrica, no planejamento das intervenções ao doente mental e suas famílias, integrados em equipes multidisciplinares, que será de grande valia não só no que diz respeito à avaliação do contexto familiar, mas também na participação da reabilitação psicossocial e continuidade de cuidados. Qualificar os cuidados de enfermagem prestados aos familiares são essenciais para a reabilitação do doente e a consequente diminuição da sobrecarga e do stress dos familiares cuidadores e se baseiam em estratégias para a adesão ao tratamento incentivando-os e ajudando-os na aproximação da família com o paciente viabilizando suas necessidades facilitando diariamente um convívio agradável e harmonioso.

\subsection{Obstáculos e Dificuldades das Famílias}

Muitas são os obstáculos e dificuldades encontrados pelas famílias quando um ente querido sofre de algum transtorno mental, ocasionando conflitos e muitas dúvidas entre todos. Muitos estudos publicados apontam questões de como lidar com o doente e como se pode fortalecer emocionalmente para poder ajudar, pois sabe-se que os desafios são constantes e devem ser superados todos os dias. 


\section{- Sobrecarga nas rotinas diárias do cuidador familiar}

Gomes; Melo (2012); Vaz (2014) e Alves et al. (2018) a família que convive com essa enfermidade, apresenta sobrecarga em suas rotinas diárias, no qual assumem as atividades domésticas com o doente, no dia a dia, fazendo com que o familiar se ausente do trabalho, dos seus compromissos sociais por direcionar sua atenção para o cuidado ao doente mental. Existência de sobrecarga familiar objetiva e subjetiva dos familiares de doentes com esquizofrenia enquanto realidade à qual os profissionais de saúde não podem ficar indiferentes e apoiar os familiares de doentes delineando intervenções de enfermagem que ajudem a minimizar o problema. Os cuidadores estão propensos ao estresse e alterações psicológicas como a depressão

\section{- Dificuldades para entender a Esquizofrenia}

Ruano; Silva; Aquino (2016); Gomes e Mello (2012) e Araújo et al. (2020) observaram que a esquizofrenia afeta quase todos os aspectos das funções psíquicas, tratando-se de uma doença crônica, de causas não muito conhecidas, representando grandes desafios para o doente, família e o sistema de saúde. Muitas são as dificuldades dos familiares que convivem com doentes esquizofrênicos para entender o distúrbio. Acolher essas famílias fornecendo informações sobre a doença estabelece impactos positivos gerando melhora para o paciente e a sua família.

Sobre esse aspecto Morais Xavier et al. (2012) relatam que os familiares têm pouco entendimento a respeito do diagnóstico da doença despertando a necessidade de procurar meios para esclarecer o mais simples possível sobre os sintomas e formas de tratamento durante os encontros entre profissionais, usuários e familiares.

Evidencia-se também que os familiares têm pouco ou nenhum conhecimento a respeito da esquizofrenia, mesmo que já tenha sido esclarecido pelo médico e os profissionais de enfermagem e por ser uma doença mental crônico degenerativa, as pessoas adoecidas têm dificuldades para realizar tarefas de autocuidado e higiene pessoal acarretando sobrecarga aos familiares cuidadores desse paciente.

São diversas as alterações na vida do indivíduo no exercício das funções como cuidador, principalmente a sobrecarga biopsicossocial, tendo como principais sinais e sintomas a ansiedade, estresse, isolamento social, fadiga, exaustão, entre outros, além do impacto causado pela diminuição da renda familiar.

As doenças mentais, de modo geral faz com que a família toda sofra intensamente com a situação do ente familiar adoecido e vivenciam vários sentimentos como culpa, angústia, aflição, entre outros. Após o impacto inicial, aos poucos a família inicia um processo de ajustamento com o intuito de equilibrar o convívio e sobrevivência de todos (Belden et al., 2012). Dessa forma, a presença da doença esquizofrenia no seio familiar com certeza irá alterar a rotina trazendo dificuldades tanto ao doente como de toda a família, otimizando o desequilíbrio de um ou mais membros. Essas mudanças podem afetar suas atividades cotidianas, uma vez que, os familiares cuidadores tendem a distanciar-se da vida sociofamiliar conforme a doença progride sobrecarregando emocionalmente suas atividades gerando diversas transformações na vida do cuidador.

Considera-se sobrecarga familiar o impacto no ambiente familiar pelo convívio com o portador esquizofrênico, no qual envolve vários aspectos, econômicos e emocionais, ao qual o familiar responsável pelo cuidado é submetido (Brasil., 2013). A sobrecarga pode ser dividida em duas vertentes; a sobrecarga objetiva que afeta a vida social do cuidador desempenhando tarefas tanto suas quanto de outra pessoa e também mudanças repentinas aos seus ideais e projetos de vida. Além disso, impõe perdas financeiras, excesso de tarefas a serem executadas no cuidado diário do paciente (De Almeida et al., 2010); e a sobrecarga subjetiva é abstrata se referindo aos sentimentos e emoções como culpa, desamparo e níveis de tristeza, sendo a percepção individual do familiar cuidador que possui e nutre em relação às suas responsabilidades durante o convívio com o ente adoecido (L. Cardoso et al., 2012).

Com isso, a responsabilidade dos cuidadores é maior e por isso torna-se importante oferecer suporte por parte dos profissionais com o objetivo de aliviar a sobrecarga familiar e fortalecer os vínculos entre a família, o usuário, a comunidade e 
a equipe. Assim, a enfermagem se faz importante visto que o modelo de atenção proposto para quem possui algum sofrimento mental segue a vertente de que as evoluções dos tratamentos ocorrem nos contextos das dinâmicas familiares.

É muito importante que a família receba suporte em serviços substitutivos em saúde mental e o enfermeiro tem papel fundamental como mediador, visto que, ainda há muita dificuldade no que diz respeito a atender a pessoa com transtorno mental em sua totalidade, incluindo dessa forma o contexto familiar em que o indivíduo se encontra inserido.

Desta maneira, a família tem uma função central na vida dos portadores de esquizofrenia. e tornam-se responsáveis pela administração das prescrições médicas e são os articuladores do cotidiano de seu familiar doente (Castro \& Furegato, 2008).

Destaca-se um fator preponderante que os integrantes da família ignoram muitas vezes, os sintomas por total desconhecimento e não aceitam a doença, fazendo com que ela se acentue cada vez mais, pelo fato de não administrar corretamente os medicamentos ora prescritos. Cabe, portanto, ao pessoal de enfermagem a responsabilidade de partilhar com os familiares, informações relevantes em função de cada caso, para os dotar de competências para lidarem com as situações dessa doença no dia-a-dia.

Valorizar a família no seu papel de cuidadora e principalmente pelo seu envolvimento no processo terapêutico é fundamental na recuperação do doente o que contribuirá para a diminuição do número de recaídas e internação dos portadores dessa doença.

Sabe-se que a relação familiar com um portador de esquizofrenia geralmente é tensa, mas se os sintomas forem controlados, o convívio pode ser harmonioso, principalmente quando os cuidadores estiveram motivados e preparados para inclui-los ativamente na sociedade por meio de tratamento eficaz e uma relação positiva com a equipe de saúde promovendo uma qualidade de vida para todos os envolvidos.

Todos os artigos analisados mostraram a importância da presença do familiar no processo de reabilitação social da pessoa portadora de esquizofrenia e sua inserção no tratamento, e a necessidade da equipe de enfermagem em se comprometer com ações de educação em saúde.

\section{Conclusão}

Ao longo da trajetória da assistência à doença mental, durante décadas sempre houve o afastamento desses doentes do convívio social e familiar. Nos dias atuais a família está diretamente envolvida no tratamento de pessoas portadoras de esquizofrenia, não sendo preciso isolá-los ou retirá-los do seio familiar e social, e os cuidados emergem da capacidade de criar novas formas de conviver com o outro, em suas diferenças.

Apesar disso, muitas famílias que convivem com portadores de esquizofrenia têm dificuldades para entender esse distúrbio ocasionando muitas dúvidas a respeito do diagnóstico da doença, despertando a necessidade de procurar meios para esclarecer de forma simples, os sintomas e formas de tratamento durante os encontros com o médico e os profissionais de enfermagem.

Com isso, a equipe de enfermagem juntamente com a equipe multidisciplinar tem papel importante na reabilitação do paciente e na orientação dos familiares cuidadores, pois é através desses profissionais que a assistência à saúde e acompanhamento ao portador serão realizados.

Evidenciou-se nesse estudo, que a produção do conhecimento científico de Enfermagem em esquizofrenia se direcionou à prestação de assistência da enfermagem no atendimento de pessoas esquizofrênicas como contribuição na relação com a família durante o processo do tratamento.

A assistência de enfermagem para tratar de pacientes esquizofrênicos deve estabelecer uma relação eficaz, sendo primordial abordar o paciente por meio de palavras diretas, claras e simples para deixá-los autoconfiantes no tratamento e a 
responsabilidade de partilhar com os familiares, informações relevantes em função de cada caso, para os dotar de competências para lidarem com as situações dessa doença no dia-a-dia.

Portanto, o apoio dos profissionais de enfermagem é imprescindível para prover condições para que os responsáveis por cuidar desse paciente sejam capazes de criar mecanismos que amparem esta prática. É fundamental valorizar a família no seu papel de cuidadora, principalmente pelo seu envolvimento no processo terapêutico na recuperação do doente para incluilos ativamente na sociedade por meio de tratamento eficiente e uma relação positiva com a equipe de saúde promoverá uma qualidade de vida para todos os envolvidos.

Conclui-se que a abordagem do enfermeiro a pacientes portadores de esquizofrenia e seus familiares é peça imprescindível no acompanhamento e tratamento, visando melhor qualidade do atendimento e tem papel fundamental como mediador.

Torna-se importante realizar novos estudos que possam contribuir com a instrumentalização e qualificação da assistência de enfermagem em direcionar os cuidados com o paciente acometido pela esquizofrenia, embora exista dificuldades próprias do transtorno, seus cuidados beneficiam não somente o paciente em tratamento, mas toda uma coletividade que faz parte da vida desse indivíduo, enfatizando a importância da relação terapêutica entre enfermeiro, paciente e familiares.

\section{Referências}

Alves, J. M., Assunção, ;, Almeida, L., Pereira Da Mata, M. A., Maria, ;, \& Pimentel, H. (2018). Problemas dos cuidadores de doentes com esquizofrenia: a sobrecarga familiar. Revista Portuguesa de Enfermagem de Saúde Mental, 19, 8-16. https://doi.org/10.19131/rpesm.0197

Araújo, V. L., Hanemann, L. D., Moura, H. S. D., Silva, A. L. C. da, Bezerra, M. de M., Silva, D. B. da, Souza, S. F. de, Antão, N. S. S., Martins, F. M., Fonseca, F. S., \& Lopes, G. de S. (2020). Convívio sociofamiliar do paciente com esquizofrenia. Research, Society and Development, 9(11), e76391110673. https://doi.org/10.33448/rsd-v9i11.10673

Belden, J. B., Hanson, B. R., McMurry, S. T., Smith, L. M., \& Haukos, D. A. (2012). Assessment of the effects of farming and conservation programs on pesticide deposition in high plains wetlands. Environmental Science and Technology, 46(6), 3424-3432. https://doi.org/10.1021/es300316q

Brasil. (2013). Saúde mental: Cadernos de atenção básica (Issue 34, p. 173). Brasil. Ministério da Saúde. Secretaria de Atenção à Saúde. Departamento de Atenção Básica. www.dab.saude.gov.br

Brasil. (2001). LEI No 10.216, DE 6 DE ABRIL DE 2001. Proteção e Os Direitos Das Pessoas Portadoras de Transtornos Mentais e Redireciona o Modelo Assistencial Em Saúde Mental. http://www.planalto.gov.br/ccivil_03/leis/leis_2001/110216.htm

Cardoso, A. M. P. (2008). O insight em psiquiatria. Fractal : Revista de Psicologia, 20(2), 347-355. https://doi.org/10.1590/s1984-02922008000200003

Cardoso, L., Galera, S. A. F., \& Vieira, M. V. (2012). O cuidador e a sobrecarga do cuidado à saúde de pacientes egressos de internação psiquiátrica. ACTA Paulista de Enfermagem, 25(4), 517-523. https://doi.org/10.1590/S0103-21002012000400006

Carniel, A. C. D. (2008). O acompanhamento terapêutico na assistência e reabilitação psicossocial do portador de transtorno mental [Biblioteca Digital de Teses e Dissertações da Universidade de São Paulo]. https://doi.org/10.11606/D.22.2008.tde-06082008-143012

Castro, S. A. de, \& Furegato, A. R. F. (2008). Conhecimento e atividades da enfermagem no cuidado do esquizofrênico. Revista Eletrônica de Enfermagem, 10(4). https://doi.org/10.5216/ree.v10.46763

D’Assunção, C. F., Santos, A. L. D. dos, Lino, F. A., \& Silveira, E. A. A. (2016). A enfermagem e o relacionamento com os cuidadores dos portadores de esquizofrenia. Rev. Enferm. Cent.-Oeste Min, 2034-2051. http://www.seer.ufsj.edu.br/index.php/recom/article/view/709

da Silva, R. C. B. (2006). Esquizofrenia: Uma revisão. In Psicologia USP (17, 263-285). Instituto de Psicologia da Universidade de São Paulo. https://doi.org/10.1590/S0103-65642006000400014

De Almeida, M. M., Schal, V. T., Martins, A. M., \& Modena, C. M. (2010). A sobrecarga de cuidadores de pacientes com esquizofrenia. Revista de Psiquiatria Do Rio Grande Do Sul, 32(3), 73-79. https://doi.org/10.1590/S0101-81082010005000003

de Andrade, F. B., Bezerra, A. I. C., de Pontes, A. L. F., Ferreira Filha, M. O., Vianna, R. P. de T., Dias, M. D., \& Silva, A. O. (2009). Saúde mental na atenção básica: um estudo epidemiológico baseado no enfoque de risco. Revista Brasileira de Enfermagem, 62(5), 675-680. https://doi.org/10.1590/s003471672009000500004

Estadão Saúde. (2020, January 23). Transtornos mentais: ansiedade cresce entre a população mundial • Summit Saúde. https://summitsaude.estadao.com.br/desafios-no-brasil/transtornos-mentais-ansiedade-cresce-entre-a-populacao/

Fortes, H. M. (2010). Tratamento compulsório e internações psiquiátricas. Revista Brasileira de Saude Materno Infantil, 10(SUPPL. 2), 10. https://doi.org/10.1590/s1519-38292010000600009 
Gomes, M. S., \& Mello, R. (2012). Sobrecarga gerada pelo convívio com o portador de esquizofrenia: enfermagem construindo o cuidado à família. Rev. Eletrônica Saúde Mental Álcool Drog., 8(1). http://pepsic.bvsalud.org/scielo.php?script=sci_arttext\&pid=S1806-69762012000100002

Holmes, D. S. (1997). Psicologia dos transtornos mentais (2nd ed.). Artmed. https://edisciplinas.usp.br/pluginfile.php/56200 54/mod_resource/content/1/David_Holmes_Psicologia_dos_transtornos.pdf

Iacoponi, E. (1999). Classificação de Transtornos Mentais e de Comportamento da CID-10 - Diretrizes Diagnósticas e de Tratamento para

Transtornos Mentais em Cuidados Primários. Revista Brasileira de Psiquiatria, 21(2), 132-132. https://doi.org/10.1590/s1516-44461999000200014

Lopes, M. H. I. (2001). Pesquisa em Hospital Psiquiátrico. https://www.ufrgs.br/bioetica/psiqpes.htm

Melo, B. B. M. De, Souza, G. D. N., \& Lopes, G. de S. (2020). Aspectos relacionados a esquizofrenia: um relato de experiência sobre a realidade do paciente e familiares. Research, Society and Development, 9(11), e60691110278. https://doi.org/10.33448/rsd-v9i11.10278

Monteiro dos Santos, A., Pimenta Marques, C., \& Bellotti de Souza, N. (2019). ESQUIZOFRENIA: assistência de Enfermagem ao paciente esquizofrênico. Revista Científica Online, 11(2).

Morais Xavier, J., Magalhães de Brito, E., Neuma Dantas Cavalcante de Abreu, R., \& Maria Magalhães Moreira, T. (2012). Percepção dos familiares de pessoas com esquizofrenia acerca da doença. Rev Bras Promoç Saúde, 25(2), 161-166. https://doi.org/10.5020/18061230.2012.2224

Oliveira, R. M., Facina, P. C. B. R., \& Siqueira Júnior, A. C. (2012). The reality of living with schizophrenia. Revista Brasileira de Enfermagem, 65(2), 309316. https://doi.org/10.1590/s0034-71672012000200017

OPAS. (2021). Transtornos mentais - OPAS/OMS | Organização Pan-Americana da Saúde. OPAS. https://www.paho.org/pt/topicos/transtornos-mentais

Pereira Lopes, W., \& Buriola, A. (2015). Esquizofrenia: conceito, epidemiologia e papel da enfermagem na adesão ao tratamento. COLLOQUIUM VITAE, 7(Especial), 81-88. https://doi.org/10.5747/cv.2015.v07.nesp.000254

Porto. (2021). psiquiatria | Definição ou significado de psiquiatria no Dicionário Infopédia de Termos Médicos. https://www.infopedia.pt/dicionarios/termosmedicos/psiquiatria

Ramminger. Tatiana. (2002). A saúde mental do trabalhador em saúde mental: um estudo com trabalhadores de um hospital psiquiátrico . Bol. Da Saúde, 16(1). http://www.boletimdasaude.rs.gov.br/conteudo/1178/a-saude-mental-do-trabalhador-em-saude-mental:-um-estudo-com-trabalhadores-de-um-hospitalpsiquiatrico

Rodriguez Belmonte, P. (1996). A reforma psiquiátrica e os novos desafios da formação de recursos humanos . FIOCRUZ.

Ruano, J. M. da Si., Silva, W. A. S., \& Aquino, R. G. (2016). Esquizofrenia: contribuição da enfermagem no tratamento e enfrentamento pelo indivíduo e família. Anais Do Fórum de Iniciação Científica Da FUNEC, 7(7). https://seer.unifunec.edu.br/index.php/forum/article/view/2516

Sade, R. M. S. (2014). Portas abertas: do manicômio ao território.

Sadock, B. J., Sadock, V. A., Ruiz, P., \& De Psiquiatria, C. (n.d.). Compêndio de Psiquiatria.

Santos, A. (2017). Papel do enfermeiro frente ao cuidado de pacientes esquizofrênicos.

Santos, P. A. N., \& Miranda, M. B. S. (2015). O percurso histórico da reforma psiquiátrica até a volta para casa . Escola de Medicina e Saúde Pública Bahiana.

Souza, J. M., \& Gusmão, L. D. (2017). Assistência de Enfermagem ao Paciente portador de Esquizofrenia: Uma Revisão Integrativa da Literatura. Id on Line REVISTA DE PSICOLOGIA, 11(38), 867-878. https://doi.org/10.14295/idonline.v11i38.934

Stefanell, M. C. (org), Fukuda, I. M. K., \& Arantes, E. C. (2008). Enfermagem psiquiátrica em suas dimensões assistenciais. Manole. https://edisciplinas.usp.br/pluginfile.php/534485/mod_resource/content/1/2sem2015/Capitulos/Stefanelli_Capitulos3.pdf

Tengan, S. K., \& Maia, A. K. (2004). Psicoses funcionais na infância e adolescência. Jornal de Pediatria, 80(2), 3-10. https://doi.org/10.1590/s002175572004000300002

Vaz, S. M. G. (2014). Os Cuidados de Enfermagem dirigidos à família, enquanto prestadora de cuidados da pessoa com esquizofrenia [Escola Superior de Enfermagem do Porto]. https://comum.rcaap.pt/bitstream/10400.26/9534/1/Tese Sónia Vaz - Documento Final.pdf

Videbeck. Sheila L. (2012). Enfermagem em Saúde Mental e Psiquiatria (5th ed.). Artmed. https://www.amazon.com.br/Enfermagem-em-Saúde-MentalPsiquiatria/dp/8536327286/ref=asc_df_8536327286/?tag=googleshopp00-20\&linkCode=df0\&hvadid=379726605826\&h vpos=\&hvnetw=g\&hvrand= $4864302051239941633 \&$ hvpone $=\& h v p t w o=\& h v q m t=\& h v d e v=c \& h v d v c m d l=\&$ hvlocint $=\&$ hvlocphy $=1031466 \&$ hvtargid=pla $-811086209168 \& \mathrm{psc}=1$ 